\title{
Q (D) Intravenous alteplase for stroke with unknown time of onset guided by advanced imaging: systematic review and meta-analysis of individual patient data
}

\begin{abstract}
Götz Thomalla, Florent Boutitie, Henry Ma, Masatoshi Koga, Peter Ringleb, Lee H Schwamm, Ona Wu, Martin Bendszus, Christopher F Bladin, Bruce C V Campbell, Bastian Cheng, Leonid Churilov, Martin Ebinger, Matthias Endres, Jochen B Fiebach, Mayumi Fukuda-Doi, Manabu Inoue, Timothy J Kleinig, Lawrence L Latour, Robin Lemmens, Christopher R Levi, Didier Leys, Kaori Miwa, Carlos A Molina, Keith W Muir, Norbert Nighoghossian, Mark W Parsons, Salvador Pedraza, Peter D Schellinger, Stefan Schwab, Claus Z Simonsen, Shlee S Song, Vincent Thijs, Danilo Toni, Chung Y Hsu, Nils Wahlgren, Haruko Yamamoto, Nawaf Yassi, Sohei Yoshimura, Steven Warach, Werner Hacke, Kazunori Toyoda, Geoffrey A Donnan, Stephen M Davis, Christian Gerloff, on behalf of the Evaluation of unknown Onset Stroke thrombolysis trials (EOS) investigators*
\end{abstract}

\section{Summary}

Lancet 2020; 396: 1574-84 Published Online November 8, 2020 https://doi.org/10.1016/ S0140-6736(20)32163-2 *EOS consortium listed in the appendix

Klinik und Poliklinik für Neurologie, Kopf- und Neurozentrum, Universitätsklinikum Hamburg-Eppendorf, Hamburg, Germany (Prof G Thomalla MD, $B$ Cheng MD, Prof ( Gerloff MD); Hospices Civils de Lyon, Service de Biostatistique, Lyon, France (F Boutitie PhD); Université Lyon 1, Villeurbanne, France (F Boutitie); Laboratoire de Biométrie et Biologie Evolutive, Equipe Biostatistique-Santé,

Villeurbanne, France (F Boutitie); Department of Medicine, School of Clinical Sciences, Monash University, Melbourne, VIC, Australia (Prof H Ma PhD); Department of

Cerebrovascular Medicine (M Koga MD, M Fukuda-Doi MD, $M$ Inoue MD, K Miwa MD, Prof K Toyoda MD,

SYoshimura MD), Department of Data Science (M Fukuda-Doi), and Center for Advancing Clinical and Translational Sciences (H Yamamoto MD), National Cerebral and

Cardiovascular Center, Suita, Japan; Department of Neurology (P Ringleb MD, Prof W Hacke MD) and Department of Neuroradiology (Prof M Bendszus MD), University of Heidelberg, Heidelberg, Germany;

Department of Neurology, Massachusetts General Hospital, Boston, MA, USA (Prof L H Schwamm MD);

Background Patients who have had a stroke with unknown time of onset have been previously excluded from thrombolysis. We aimed to establish whether intravenous alteplase is safe and effective in such patients when salvageable tissue has been identified with imaging biomarkers.

Methods We did a systematic review and meta-analysis of individual patient data for trials published before Sept 21, 2020. Randomised trials of intravenous alteplase versus standard of care or placebo in adults with stroke with unknown time of onset with perfusion-diffusion MRI, perfusion CT, or MRI with diffusion weighted imaging-fluid attenuated inversion recovery (DWI-FLAIR) mismatch were eligible. The primary outcome was favourable functional outcome (score of $\mathbf{0 - 1}$ on the modified Rankin Scale [mRS]) at $\mathbf{9 0}$ days indicating no disability using an unconditional mixed-effect logistic-regression model fitted to estimate the treatment effect. Secondary outcomes were mRS shift towards a better functional outcome and independent outcome (mRS 0-2) at 90 days. Safety outcomes included death, severe disability or death (mRS score 4-6), and symptomatic intracranial haemorrhage. This study is registered with PROSPERO, CRD42020166903.

Findings Of 249 identified abstracts, four trials met our eligibility criteria for inclusion: WAKE-UP, EXTEND, THAWS, and ECASS-4. The four trials provided individual patient data for 843 individuals, of whom $429(51 \%)$ were assigned to alteplase and $414(49 \%)$ to placebo or standard care. A favourable outcome occurred in $199(47 \%)$ of 420 patients with alteplase and in 160 (39\%) of 409 patients among controls (adjusted odds ratio [OR] 1.49 [95\% CI 1.10-2.03]; $\mathrm{p}=\mathbf{0} \cdot \mathbf{0 1 1})$, with low heterogeneity across studies $\left(I^{2}=27 \%\right)$. Alteplase was associated with a significant shift towards better functional outcome (adjusted common OR 1.38 [95\% CI 1.05-1.80]; $\mathrm{p}=\mathbf{0} \cdot 019$ ), and a higher odds of independent outcome (adjusted OR 1.50 [1.06-2.12]; $\mathrm{p}=0.022$ ). In the alteplase group, $90(21 \%)$ patients were severely disabled or died (mRS score 4-6), compared with 102 (25\%) patients in the control group (adjusted OR 0.76 [0.52-1.11]; $\mathrm{p}=0 \cdot 15) .27(6 \%)$ patients died in the alteplase group and $14(3 \%)$ patients died among controls (adjusted OR $2.06[1.03-4.09] ; p=0.040)$. The prevalence of symptomatic intracranial haemorrhage was higher in the alteplase group than among controls (11 [3\%] vs two [<1\%], adjusted OR $5 \cdot 58$ [1·22-25.50]; $\mathrm{p}=0 \cdot 024)$.

Interpretation In patients who have had a stroke with unknown time of onset with a DWI-FLAIR or perfusion mismatch, intravenous alteplase resulted in better functional outcome at 90 days than placebo or standard care. A net benefit was observed for all functional outcomes despite an increased risk of symptomatic intracranial haemorrhage. Although there were more deaths with alteplase than placebo, there were fewer cases of severe disability or death.

Funding None.

Copyright @ 2020 Elsevier Ltd. All rights reserved.
Introduction

Intravenous thrombolysis with alteplase is standard care for acute ischaemic stroke. This treatment improves functional outcomes and is more effective the earlier treatment is initiated. ${ }^{1}$ Since its first approval for stroke treatment, intravenous alteplase has been restricted to patients with known time of symptom onset within a narrow time window. This window was initially within less than $3 \mathrm{~h}$ of symptom onset based on the results of the NINDS trial ${ }^{2}$ and was extended to a time window of less than $4.5 \mathrm{~h}$ following the positive results of ECASS-3 and a subsequent meta-analysis. ${ }^{1.4}$ Patients with unknown 
Research in context

\section{Evidence before this study}

To establish the need for this meta-analysis, we searched PubMed for meta-analyses of alteplase for stroke in unknown onset or wake-up stroke published up until Sept 21, 2020, in English. This search only yielded two meta-analyses, of which only one included randomised trials, but did not represent an individual patient data meta-analysis. Therefore, we did a systematic review for randomised trials of intravenous alteplase versus standard of care or placebo in adults with acute ischaemic stroke and unknown time of symptom onset using advanced brain imaging with either penumbral imaging (ie, perfusion-diffusion MRI or perfusion CT) or MRI-based tissue-clocking (ie, a mismatch on MRI between a visible lesion on diffusion weighted imaging [DWI] and no marked parenchymal hyperintensity on fluid attenuated inversion recovery [FLAIR]) with more than 20 patients. We included studies published from database inception until Sept 21, 2020. We identified four trials. The WAKE-UP trial randomly assigned 503 patients with unknown time of onset stroke to intravenous alteplase or placebo if they had DWI-FLAIR mismatch in visual assessment and found a significantly better functional outcome in patients given alteplase than placebo. The EXTEND trial randomly assigned 225 patients with late time window or unknown time of onset stroke to alteplase after automated CT or MRI perfusion imaging and showed higher rates of excellent functional outcome (defined as a score of $0-1$ on the modified Rankin Scale [mRS] at 3 months) compared with placebo. The THAWS trial randomly assigned 131 patients with unknown time of onset stroke to treatment with alteplase or standard of care on the basis of visual assessment of the DWI-FLAIR mismatch and reported neutral outcomes, but was underpowered. The ECASS-4 trial randomly assigned 119 patients with late time window or unknown time of onset stroke and penumbral mismatch on perfusion-diffusion MRI and reported neutral outcomes, but was also underpowered.
All four studies were stopped early for different reasons, and had only modest sample sizes limiting strength and precision of the findings.

\section{Added value of this study}

This systematic review and individual patient data metaanalysis of four randomised trials quantifies the benefits and risks of intravenous alteplase for patients with stroke with unknown time of onset. Intravenous alteplase resulted in higher rates of excellent functional outcome defined as a score of $0-1$ on the $\mathrm{mRS}$ at 90 days than placebo or standard care. A net benefit was observed for all functional outcomes across the entire range of the $\mathrm{mRS}$ despite an increased risk of symptomatic intracranial haemorrhage. Although there was a higher prevalence of death with alteplase, dependence or death (mRS 3-6) was less common than in the control group. Subgroup analysis did not identify a significant treatment heterogeneity in relevant subgroups but confirmed a consistent treatment effect across a wide range of subgroups. We also observed a significant treatment benefit in the subgroup of patients with a large vessel occlusion.

\section{Implications of all the available evidence}

Patients with stroke with unknown time of symptom onset, DWI-FLAIR mismatch or perfusion mismatch, who received treatment with intravenous alteplase had a better functional outcome at 90 days than patients receiving placebo or standard of care. There was a net benefit for all functional outcomes with alteplase and also similar frequencies of severe disability or death in both groups, despite an increased risk of symptomatic These results extend the findings from individual trials and provide level 1a evidence for the use of brain imaging beyond non-contrast CT to guide treatment with intravenous alteplase in patients with stroke with an unknown time of onset. intracranial haemorrhage and a higher mortality with alteplase.

Athinoula A Martinos Center for Biomedical Imaging, Department of Radiology, Massachusetts General Hospital, Charlestown, MA, USA (O Wu PhD); Department of Neurosciences, Eastern Health and Eastern Health Clinical School, Monash University, Box Hill, VIC, Australia (Prof C F Bladin MD); Departments of Medicine and Neurology, Melbourne Brain Centre at the Royal Melbourn Hospital, The University of Melbourne, Melbourne, VIC, Australia (Prof L Churilov PhD, Prof B C V Campbell PhD, Prof M W Parsons PhD, NYassi PhD,

Prof GA Donnan MD,

Prof S M Davis MD); Centrum für Schlaganfallforschung Berlin (Prof M Ebinger MD, Prof M Endres MD, Prof J B Fiebach MD) and Klinik und Hochschulambulanz fü Neurologie (Prof M Endres), Charité-Universitätsmedizin Berlin, Berlin, Germany; Klinik für Neurologie Medical Park Berlin Humboldtmühle, Berlin Germany (Prof M Ebinger); German Centre of

Cardiovascular Research, Berlin, Germany (Prof M Endres); German Center of Neurodegenerative Diseases, Berlin, Germany (Prof M Endres); Department of Neurology, Royal Adelaide Hospital, Adelaide, SA, Australia (ProfT J Kleinig PhD); Acute Cerebrovascular Diagnostics Unit, National Institute of Neurological Disorders and Stroke, National Institutes of Health, Bethesda, MD, USA (L L Latour PhD);

(FLAIR) on MRI (termed DWI-FLAIR mismatch). ${ }^{6}$ The WAKE-UP trial provided evidence of benefit of treatmen with intravenous alteplase in patients with stroke with unknown time of onset if the treatment decision was based on DWI-FLAIR mismatch. ${ }^{7}$ Current US and European guidelines and consensus statements recommend intravenous thrombolysis with alteplase in patients with unknown time of symptom onset if patients meet the WAKE-UP criteria., ${ }^{8,9}$ On the basis of MR or CT perfusion imaging, the EXTEND trial also showed better functional outcomes in alteplase-treated patients awakening with stroke or treated within 4.5 to $9.0 \mathrm{~h}$ after symptom onset. ${ }^{10}$

Both WAKE-UP and EXTEND were started before compelling evidence for stroke thrombectomy was available and both excluded patients in whom throm bectomy was planned. In the meantime, two trials of endovascular stroke treatment, DAWN and DEFUSE-3,
Department of Neurosciences, Experimental Neurology, KU Leuven, University of Leuven, Leuven, Belgium (R Lemmens MD); VIB Center for Brain and Disease Research Leuven, Belgium (R Lemmens); Department of Neurology, University Hospitals Leuven, Leuven, Belgium (R Lemmens); The Department of Neurology, Priority Research Centre for Brain and Mental Health Research, John Hunter Hospital, University of Newcastle, Newcastle, NSW, Australia (Prof C R Levi MD); Université de Lille, Inserm U1171, Lille, France (Prof D Leys MD); Stroke Unit, Department of Neurology, Vall d'Hebron, Barcelona, Spain Hospital Universitari 
(Prof C A Molina MD); Institute of Neuroscience and Psychology, University of Glasgow, Glasgow, UK (Prof K W Muir MD); Department of Stroke Medicine, Université Claude Bernard Lyon 1, CarMeN Laboratory, INSERM U1060/ INRA 1397, Lyon, France; (Prof N Nighoghossian MD); Department of Radiology, Institut de Diagnòstic per la Imatge, Hospital Dr Josep Trueta, Institut d'Investigació Biomèdica de Girona, Girona, Spain (S Pedraza PhD); Universitätsklinik für Neurologie,

Mühlenkreiskliniken, Johannes Wesling Klinikum Minden, Universitätsklinikum der Ruhr-Universität Bochum, Minden, Germany (Prof P D Schellinger MD); Department of Neurology, University of ErlangenNuremberg, Erlangen, Germany (Prof S Schwab MD); Department of Neurology, Aarhus University Hospital, Aarhus, Denmark

(CZ Simonsen MD); Department of Neurology, Cedars-Sinai Medical Center, Los Angeles, CA, USA (S S Song MD); Florey Institute of Neuroscience and Mental Health, Heidelberg, VIC, Australia (Prof V Thijs PhD); Department of Neurology, Austin Health, Heidelberg, VIC, Australia (Prof V Thijs); Department of Human Neurosciences, University of Rome "La Sapienza", Rome, Italy (Prof D Toni MD); Graduate Institute of Clinical Medical Science, China Medical University, Taichung, Taiwan (Prof CY Hsu PhD); Department of Clinical Neuroscience, Karolinska Institutet, Stockholm, Sweden (Prof N Wahlgren MD); Population Health and Immunity Division, The Walter and Eliza Hall Institute of

Medical Research, Parkville, VIC, Australia (N Yassi); and Dell Medical School, University of Texas at Austin, Austin, TX, USA (Prof S Warach MD)

Correspondence to:

Prof Götz Thomalla, Kopf- und Neurozentrum, Klinik und Poliklinik für Neurologie, Universitätsklinikum HamburgEppendorf, D-20246 Hamburg, Germany

thomalla@uke.de See Online for appendix provided evidence of a benefit of mechanical thrombectomy guided by penumbral imaging in a late or unknown time window. ${ }^{11,12}$

Nevertheless, the individual trials were still modest in size and evidence is scarce for the efficacy and safety of thrombolysis in subgroups of patients with an unknown time of stroke onset. An individual patient data metaanalysis from 2019 of the trials using penumbral imaging to guide intravenous thrombolysis in patients in an extended or unknown time window (EXTEND, ECASS-4, and EPITHET) indicated that intravenous alteplase improved functional outcome in these patients $(n=414)$, with an overall net clinical benefit despite an increase in the rate of symptomatic intracranial haemorrhage and a numerical but non-significant increase in deaths in the thrombolysis group. ${ }^{13}$ We aimed to establish whether intravenous alteplase was safe and effective in patients with stroke with an unknown time of onset when salvageable tissue is identified with imaging biomarkers. To this end, we did a systematic review and meta-analysis of individual patient data $(n=843)$. We tested the hypothesis that intravenous alteplase improves functional outcomes compared with placebo or standard care in patients with acute ischaemic stroke with an unknown time of onset if selected with imaging biomarkers (ie either DWI-FLAIR mismatch or CT or MRI based penumbral imaging).

\section{Methods}

\section{Search strategy and selection criteria}

For the systematic review, we searched PubMed, Web of Science, SciELO, and LILACS for clinical trials published in English from database inception up until Sept 21, 2020, with the following search terms: "stroke" AND ("alteplase" OR "rtPA" OR "tPA" OR "thrombolysis") AND ("randomised" OR "randomized”) AND ("unknown” OR "unwitnessed” OR “wake-up” OR "extended") (filters activated: "Clinical trial", "Humans") Studies that were eligible for inclusion were: randomised trials of intravenous alteplase versus standard of care or placebo in adults ( $\geq 18$ years), with acute ischaemic stroke and unknown time of symptom onset, using advanced brain imaging with either penumbral imaging (ie, perfusion-diffusion MRI or perfusion CT) or MRIbased tissue-clocking (ie, DWI-FLAIR mismatch), and with more than 20 patients enrolled. We also searched ClinicalTrials.gov, the EU Clinical Trials Register, the WHO International Clinical Trials Registry Platform, the ISRCTN Registry, and the Cochrane Central Register of Controlled Trials for clinical trials of intravenous alteplase in stroke with an unknown time of onset. All patients included in the primary analyses of individual trials were considered eligible for inclusion in the metaanalysis. Two reviewers (BC and GT) independently reviewed articles and reached a unanimous decision for inclusion. In cases of potential conflicts, discussions with members of the evaluation of unknown onset stroke thrombolysis trials (EOS) steering committee were planned. There was, however, no conflict in the judgment of the results between both reviewers based on the predefined criteria for inclusion in the metaanalysis.

The steering committees of all included trials agreed to join the EOS collaboration and share individual patientlevel data for the meta-analysis. Ethical approval was obtained for all participating sites for all included trials and patients or their legal representatives provided written informed consent according to national and local regulations including an exception from explicit informed consent in emergency circumstances in some countries. The protocol for this study was prespecified and followed PRISMA guidelines for meta-analyses of individual patient data (appendix pp 31-40). The study is registered with PROSPERO, number CRD42020166903).

\section{Outcomes}

The prespecified primary outcome was a favourable outcome defined by a score of $0-1$ on the modified Rankin Scale (mRS; which ranges from 0 [no symptoms] to 6 [death]) at 90 days after stroke. This cutoff identifies patients with no symptoms at all (mRS score 0 ) or only minimal symptoms with no significant disability, being able to do all usual activities (mRS score 1). Secondary outcomes were functional improvement across the entire mRS (ie, mRS shift analysis) at 90 days and independent outcome defined by a score of $0-2$ on the mRS at 90 days. Safety outcomes were death, severe disability or death (ie, mRS score 4-6), symptomatic intracranial haemorrhage according to the Safe Implementation of Thrombolysis in Stroke Monitoring Study $\left(\right.$ SITS-MOST) ${ }^{14}$ and radiologically defined parenchyma haemorrhage type $2 .{ }^{15}$ Additional outcomes were death within 7 days of randomisation and death or dependence (ie, MRS score 3-6) at 90 days.

We re-analysed all available imaging data as follows. Judgment of vessel occlusion was on the basis of image readings provided by the individual trials. Any vessel occlusion was defined as any visible occlusion of an intracranial brain supplying artery on baseline MR-angiography or CT-angiography. Large vessel occlusion was defined as occlusion of the intracranial internal carotid artery or main stem of the middle cerebral artery. Penumbral mismatch was defined according to the criteria used in studies published in the past 3 years of intravenous alteplase on the basis of perfusion mismatch. ${ }^{13}$ Penumbral mismatch was considered present with a mismatch ratio between critically hypoperfused tissue and infarct core of more than $1 \cdot 2$, a mismatch volume greater than $10 \mathrm{~mL}$, and an ischaemic core volume less than $70 \mathrm{~mL}$. Critically hypoperfused tissue was defined as tissue with a time to maximum of the residue function of more than $6 \mathrm{~s}$ in CT perfusion or MR perfusion. Infarct core was defined as a relative cerebral blood flow of less than $30 \%$ of contralateral 
cerebral blood flow for CT perfusion or an apparent diffusion coefficient of less than $620 \mu \mathrm{m}^{2} / \mathrm{s}$ for diffusion MRI. CT perfusion, MR perfusion, and DWI data, if available, were reprocessed using automated processing software RAPID (version 4.6, 4.9, and 5.0) as described previously. ${ }^{11,12}$ DWI-FLAIR mismatch was defined according to the criteria used in the WAKE-UP trial-ie, a mismatch between an acute ischaemic lesion visible on DWI and no marked parenchymal hyperintensity on FLAIR in the corresponding region as assessed by visual inspection. ${ }^{16}$

\section{Data analysis}

The full statistical analysis plan is provided in the appendix (pp 31-40). The responsible statisticians or trial coordinators (FB, LC, PR, MI, OW) from each trial extracted the patient-level data from the trial databases on the basis of data fields prespecified in the study protocol (appendix pp 35-36). The biostatistician responsible for the meta-analysis (FB) collated all data from the individual trials and cross-checked them against the original publications of the individual trials.

The modified Cochrane Collaboration tool to assess risk of bias for randomised controlled trials was applied for qualitative assessment of between trial differences including patient eligibility and assessment of bias (appendix pp 26-27).

This meta-analysis followed a one-stage approach with the use of relevant mixed-effect logistic regression models with trial as a random intercept effect and treatment assignment as a random slope effect, allowing the treatment effect to vary across trials. As randomisation in the WAKE-UP trial was stratified by age ( $\leq 60$ years or $>60$ years) and severity of symptoms (National Institute of Health Stroke Scale [NIHSS] $\leq 10$ or $>10$ ) at baseline, all models were adjusted on these two categorical covariates. All outcomes were assessed in the intentionto-treat population (ie, all patients randomly assigned to their randomised treatment group).

The primary efficacy outcome was analysed with use of an unconditional mixed-effect logistic-regression model fitted to estimate the treatment effect, reported as an odds ratio (OR) and 95\% CI. Missing primary outcome values were replaced using multiple imputation, including the following covariates: allocated treatment, baseline age (continuous), baseline NIHSS score, and NIHSS score at day 7. We also did a sensitivity analysis without replacement of missing outcomes. The heterogeneity of treatment effect across trials was tested using the Cochran's Q-statistic. We also report the $I^{2}$ statistic describing the percentage of variation across studies that is due to heterogeneity rather than chance. ${ }^{17}$

The categorical shift in the distribution of $\mathrm{mRS}$ scores between the two treatment groups was analysed fitting a proportional-odds logistic regression model, assuming a common OR across all cut points of the mRS with values 5 and 6 collapsed into one category to prevent interpreting $\mathrm{mRS} 5$ as a better outcome than mRS 6. mRS 5 is bedriddenness and nursing home outcome with full dependence on care, which is not usually considered as a better quality of life outcome than death in stroke treatment studies. Before ordinal regression analysis, we tested the proportional odds assumption. Analysis of independent outcome, death, and severe disability or death was done with use of the same unconditional mixed-effect logisticregression model as for the primary outcome. Symptomatic intracranial haemorrhage and parenchymal haemorrhage type 2 were analysed with two-by-two tables stratified by trials and the common Cochran-Mantel-Haenszel OR and its $95 \%$ CI were calculated. The Breslow-Day test was used to test homogeneity of ORs across trials. For secondary and safety outcomes, missing values were not replaced.

We did pre-planned analyses in the following subgroups: use of standard dose $(0.9 \mathrm{mg} / \mathrm{kg}$ bodyweight) versus low dose $(0.6 \mathrm{mg} / \mathrm{kg}$ bodyweight) alteplase; age ( $\leq 60$ years $v s>60$ years); sex; baseline stroke severity (NIHSS $\leq 10 v s>10$ ); any visualised vessel occlusion; large vessel occlusion; imaging modality (CT vs MRI); history of atrial fibrillation; history of stroke or transient ischaemic attack; previous antiplatelet use; delay from symptom recognition to treatment ( $\leq 3 \mathrm{~h} v s>3 \mathrm{~h}$ ); penumbral mismatch present; and DWI-FLAIR mismatch present. For all subgroup analyses, the same model was used as for the analysis of the primary outcome, with an additional interaction parameter between the treatment and the subgroup covariate entered as a fixed effect.

In an additional analysis, we included available data for outcome and safety of intravenous alteplase in unknown onset stroke from single-arm studies.

All analyses were done with a two-sided $\alpha$ level of $0 \cdot 05$. There was no correction of the $\alpha$ level for multiple comparisons. Statistical analysis was done using SAS, version 9.4 and $R$, version 3.3.2.

\section{Role of the funding source}

There was no funding source for this study. The funders of the trials included in this meta-analysis had no role in study design, data collection, data analysis, data interpretation, or writing of this report. The corresponding author had full access to all the data in the study and had final responsibility for the decision to submit for publication.

\section{Results}

The original search identified 249 abstracts. Four studies met our eligibility criteria for inclusion: WAKE-UP, EXTEND, ${ }^{10}$ THAWS ${ }^{18}$ and ECASS $-4^{19}$ (for the PRISMA individual patient data flow diagram see appendix $\mathrm{p} 20$ ). All four trials applied brain imaging beyond non-contrast CT to randomly assign either exclusively patients with stroke with unknown time of onset, or patients with stroke within an extended time window beyond $4.5 \mathrm{~h}$ of symptom onset including unknown onset, to intravenous alteplase or placebo or standard of care. 
WAKE-UP was a European randomised, placebocontrolled trial performed in Austria, Belgium, Denmark, France, Germany, Netherlands, Spain, and the UK that used MRI with DWI-FLAIR mismatch to guide standard dose intravenous alteplase in patients with stroke with unknown time of onset. ${ }^{7}$ Although not mandatory, MR perfusion data were acquired in a subgroup of patients who were randomly assigned and available for assessment of penumbral mismatch. EXTEND was a trial in Australia, Finland, New Zealand, and Taiwan, which used penumbral imaging with either CT perfusion or perfusion-diffusion MRI to randomly assign patients in an extended time window of 4.5-9.0 h of stroke or wake-up stroke (if the midpoint between the time last known to be well and time of waking up with symptoms was within $9 \mathrm{~h}$ ) to standard

\begin{tabular}{|c|c|c|}
\hline & Alteplase group ( $n=429$ ) & Control group $(n=414)$ \\
\hline Mean age, years & $68 \cdot 5(12 \cdot 2)$ & $68 \cdot 5(12 \cdot 7)$ \\
\hline \multicolumn{3}{|l|}{ Sex } \\
\hline Men & $268(62 \%)$ & $253(61 \%)$ \\
\hline Women & $161(38 \%)$ & $161(39 \%)$ \\
\hline \multicolumn{3}{|l|}{ Reason for unknown time of symptom onset } \\
\hline Overnight sleep & $385(90 \%)$ & $366(88 \%)$ \\
\hline Other & $44(10 \%)$ & $48(12 \%)$ \\
\hline $\begin{array}{l}\text { Median time between last seen well and } \\
\text { symptom recognition, } \mathrm{h}\end{array}$ & $7 \cdot 0(4 \cdot 7-9 \cdot 0)$ & $7 \cdot 0(5 \cdot 0-9 \cdot 0)$ \\
\hline \multicolumn{3}{|l|}{ Medical history } \\
\hline Arterial hypertension & $259 / 428(61 \%)$ & $245 / 412(59 \%)$ \\
\hline Diabetes & $83 / 424(20 \%)$ & $69 / 413(17 \%)$ \\
\hline Hypercholesterolaemia* & $116 / 311(37 \%)$ & $108 / 301(36 \%)$ \\
\hline Atrial fibrillation & $86 / 427(20 \%)$ & $72 / 408(18 \%)$ \\
\hline $\begin{array}{l}\text { History of ischaemic stroke or transient } \\
\text { ischaemic attack* }\end{array}$ & $45 / 323(14 \%)$ & $45 / 310(15 \%)$ \\
\hline Pretreatment with antiplatelets & $125 / 397(31 \%)$ & $132 / 383(34 \%)$ \\
\hline Median NIHSS score & $7(4-12)$ & $7(4-12)$ \\
\hline \multicolumn{3}{|l|}{ Imaging modality } \\
\hline $\mathrm{CT}$ & $65(15 \%)$ & $64(15 \%)$ \\
\hline MRI & $364(85 \%)$ & $350(85 \%)$ \\
\hline \multicolumn{3}{|c|}{ Vessel occlusion on baseline CT-angiography or MR-angiography ${ }^{\dagger}$} \\
\hline Any vessel occlusion & $174 / 391(45 \%)$ & $168 / 380(44 \%)$ \\
\hline Large vessel occlusion & $99 / 391(25 \%)$ & $90 / 380(24 \%)$ \\
\hline \multicolumn{3}{|l|}{ Mismatch present } \\
\hline Penumbral & $114 / 214(53 \%)$ & $109 / 199(55 \%)$ \\
\hline DWI-FLAIR & $335 / 353(95 \%)$ & $320 / 339(94 \%)$ \\
\hline \multicolumn{3}{|l|}{ Alteplase dose, $\mathrm{mg} / \mathrm{kg}$ bodyweight } \\
\hline 0.9 & $359(84 \%)$ & $353(85 \%)$ \\
\hline 0.6 & $70(16 \%)$ & $61(15 \%)$ \\
\hline $\begin{array}{l}\text { Median time from symptom recognition to } \\
\text { treatment initiation, } \mathrm{h}\end{array}$ & $3 \cdot 3(2 \cdot 6-4 \cdot 1)$ & $3 \cdot 4(2 \cdot 7-4 \cdot 1)$ \\
\hline $\begin{array}{l}\text { Median time between last seen well and } \\
\text { treatment initiation, } \mathrm{h}\end{array}$ & $10 \cdot 6(8 \cdot 6-12 \cdot 4)$ & $10 \cdot 5(8 \cdot 4-12 \cdot 3)$ \\
\hline \multicolumn{3}{|c|}{$\begin{array}{l}\text { Data are mean (SD), } \mathrm{n}(\%) \text {, or median (IQR). NIHSS=National Institutes of Health Stroke Scale. DWI-FLAIR=diffusion } \\
\text { weighted imaging-fluid attenuated inversion recovery. }{ }^{*} \text { Not recorded in ECASS- } 4{ }^{19} \text { and EXTEND }{ }^{10} .+ \text { Not available fo } \\
\text { ECASS-4. }\end{array}$} \\
\hline
\end{tabular}

dose alteplase or placebo. ${ }^{10}$ THAWS was a Japanese trial using MRI with DWI-FLAIR mismatch according to WAKE-UP to randomly assign patients to a reduced dose of $0.6 \mathrm{mg} / \mathrm{kg}$ alteplase (ie, the approved dose in Japan) ${ }^{20}$ or standard of care. ${ }^{18}$ ECASS-4 was a European trial performed in Austria, Czech Republic, France, Germany, Italy, Spain, and the UK, which applied the same eligibility criteria as EXTEND but used only perfusion-diffusion MRI (not CT) to randomly assign patients to standard dose alteplase or placebo. ${ }^{19}$ ECASS-4 data were also available for assessment of DWI-FLAIR mismatch. WAKE-UP was terminated early due to cessation of funding. EXTEND and THAWS were stopped early after publication of the positive results of the WAKE-UP trial due to lack of equipoise, and ECASS-4 was terminated early due to reduced recruitment following the publication of the positive trials of thrombectomy in an extended time window. ${ }^{11,12}$ These four trials were included in the primary analysis. Importantly, from EXTEND and ECASS-4, we only included patients with unknown-onset stroke for this meta-analysis. Overall, the risk of bias in the studies included in the meta-analysis was considered low (appendix p 27). Baseline characteristics of individual trials are shown in the appendix (p 28). Our search strategy identified another study, MR WITNESS ${ }^{21}$ which was not a randomised clinical trial but met other eligibility criteria. We included this trial in a supplementary safety analysis.

We obtained data from all 843 participants with stroke with unknown time of onset, who were randomly assigned in the four trials included in the primary analysis. Of these, 429 (51\%) were assigned to receive alteplase and 414 (49\%) to receive placebo or standard of care (control group). Baseline characteristics were balanced between the groups (table 1 ).

Mean age was $68 \cdot 5$ years (SD 12.5), 322 (38\%) were women. Waking from overnight sleep was the reason for unknown symptom onset in 751 patients (89\%). Median time from last seen well to symptom recognition was 7.0 h (IQR 5.0-9·0). Median NIHSS on admission was 7 (IQR 4-12). MRI was used for randomisation in 714 (85\%) patients. A DWI-FLAIR mismatch was present in 655 (95\%) of 692 patients in whom assessment of DWI-FLAIR mismatch was done. Penumbral mismatch was present in 223 (54\%) of 413 patients with available assessment for perfusion imaging. Any vessel occlusion was present in 342 (44\%) of 771 patients in whom information on vessel status was available, and $189(25 \%)$ of them had a large vessel occlusion.

Primary outcome data were available for 829 (98\%) of 843 patients included in the analysis (420 [98\%] of 429 patients in the alteplase group and 409 [99\%] of 414 patients in the control group). We used three covariates to impute the missing primary outcome in 14 patients, two were stratification variables (age and NIHSS score at baseline) with no missing data, and one was NIHSS score at $72 \mathrm{~h}$, for which 36 missing values were imputed. 


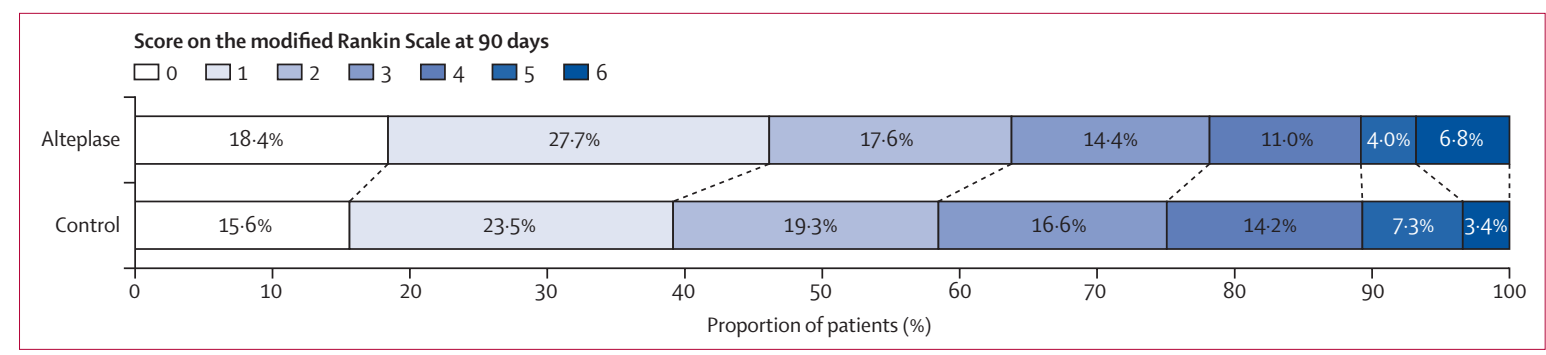

Figure 1: Distribution of scores on the modified Rankin Scale at 90 days

Scores on the modified Rankin Scale range from 0 to 6, with 0 indicating no symptoms, 1 no clinically significant disability, 2 slight disability, 3 moderate disability, 4 moderately severe disability, 5 severe disability, and 6 death.

A favourable outcome (mRS score $0-1)$ at 90 days was observed in 199 (47\%) of 420 patients in the alteplase group and in 160 (39\%) of 409 patients in the control group (adjusted OR 1.49 [95\% CI 1.10-2.03]; $\mathrm{p}=0 \cdot 011$; figure 1, table 2), with low heterogeneity across studies $\left(I^{2}=27 \%\right)$. This result represents an absolute increase of $8 \%$ of patients with favourable outcome corresponding to a number-needed-to-treat of 12 .

Treatment with alteplase was associated with a significant shift towards better functional outcome-ie, lower scores on the mRS at 90 days in ordinal analysis (adjusted common OR 1.38 [95\% CI 1.05-1.80]; $\mathrm{p}=0.019)$. The proportion of patients who reached functional independence (mRS score $0-2$ ) at 90 days was also significantly higher in the alteplase group than in the control group (adjusted OR 1.50 [95\% CI 1.06-2.12]; $\mathrm{p}=0 \cdot 022$ ). Sensitivity analysis without replacement of primary outcome confirmed a significant benefit of alteplase on the primary endpoint (appendix p 18).

At 90 days, death was reported in $27(6 \%)$ patients in the alteplase group compared with 14 patients $(3 \%)$ in the control group (adjusted OR 2.06 [95\% CI 1.03-4.09]; $\mathrm{p}=0 \cdot 040$ ). Of the 27 deaths in the alteplase group, seven were attributable to symptomatic intracranial haemorrhage, four to recurrent or progressive stroke, two were of unknown cause, and the remaining 14 deaths were of non-neurological cause and unrelated to treatment or index stroke. In the control group, all 14 deaths were of non-neurological cause and unrelated to treatment or index stroke. Death within 7 days occurred in ten $(2 \%)$ patients in the alteplase group and in four (1\%) patients in the control group (adjusted OR 2.54 [95\% CI $0 \cdot 78-8 \cdot 32] ; p=0 \cdot 19)$. In the alteplase group, $90(21 \%)$ patients were severely disabled or had died (mRS score 4-6), which was one of the safety endpoints, compared with $102(25 \%)$ patients in the control group (adjusted OR 0.76 [95\% CI $0 \cdot 52-1 \cdot 11] ; \mathrm{p}=0 \cdot 15$ ). The proportion of patients who were dependent or had died (mRS score 3-6) at 90 days was lower with alteplase than in controls (adjusted OR 0.67 [95\% CI 0.47-0.94]; $\mathrm{p}=0.022$ ). The number of patients with symptomatic intracranial haemorrhage was higher in the alteplase group than in the control group $(11[3 \%]$ vs two $[<1 \%]$,

\begin{tabular}{|c|c|c|c|c|}
\hline & $\begin{array}{l}\text { Alteplase group } \\
(\mathrm{n}=429)\end{array}$ & $\begin{array}{l}\text { Control group } \\
(n=414)\end{array}$ & $\begin{array}{l}\text { Adjusted odds ratio } \\
(95 \% \mathrm{Cl})^{*}\end{array}$ & $\mathrm{p}$ value \\
\hline \multicolumn{5}{|l|}{ Primary efficacy outcome } \\
\hline $\begin{array}{l}\text { Favourable outcome (mRS 0-1) at } \\
90 \text { days } \dagger\end{array}$ & $199 / 420(47 \%)$ & $160 / 409(39 \%)$ & $1.49(1.10-2.03)$ & 0.011 \\
\hline \multicolumn{5}{|l|}{ Secondary efficacy outcomes } \\
\hline mRS score at 90 days & .. & .. & $1.38(1.05-1 \cdot 80)$ & 0.019 \\
\hline $\begin{array}{l}\text { Independent outcome (mRS 0-2) at } \\
90 \text { days } \dagger\end{array}$ & $273 / 420(65 \%)$ & $239 / 409(58 \%)$ & $1.50(1.06-2.12)$ & 0.022 \\
\hline \multicolumn{5}{|l|}{ Safety outcomes } \\
\hline Death at 90 days $\dagger$ & $27(6 \%)$ & $14(3 \%)$ & $2.06(1.03-4.09)$ & 0.040 \\
\hline Death at 7 days & $10(2 \%)$ & $4(1 \%)$ & $2.54(0.78-8 \cdot 32)$ & $0 \cdot 19$ \\
\hline $\begin{array}{l}\text { Severe disability or death (mRS 4-6) } \\
\text { at } 90 \text { days } \dagger\end{array}$ & $90 / 420(21 \%)$ & $102 / 409(25 \%)$ & $0.76(0.52-1.11)$ & 0.15 \\
\hline $\begin{array}{l}\text { Dependence or death (mRS 3-6) at } \\
90 \text { days } \dagger\end{array}$ & $147 / 420(35 \%)$ & $170 / 409(42 \%)$ & $0.67(0.47-0.94)$ & 0.022 \\
\hline $\begin{array}{l}\text { Symptomatic intracranial } \\
\text { haemorrhage }\end{array}$ & $11(3 \%)$ & $2(<1 \%)$ & $5 \cdot 58(1 \cdot 22-25 \cdot 50)$ & 0.024 \\
\hline Parenchymal haemorrhage type $2 \ddagger$ & $11(3 \%)$ & $3(1 \%)$ & $3 \cdot 51(0.98-12 \cdot 60)$ & 0.068 \\
\hline \multicolumn{5}{|c|}{$\begin{array}{l}\text { Data are } \mathrm{n}(\%) \text { or } \mathrm{n} / \mathrm{N}(\%) \text {, unless specified. } \mathrm{mRS}=\text { modified Rankin Scale. *Odds ratios were adjusted for age and } \\
\text { symptom severity at baseline. } † \text { Numbers are given for patients with available data for the primary efficacy endpoint; } \\
\mathrm{mRS} \text { at day } 90 \text { was missing for nine patients in the alteplase group and five patients in the control group; missing } \\
\text { primary outcome values were replaced using multiple imputation. } ¥ \text { Radiological assessment of parenchymal } \\
\text { haemorrhage type } 2 \text { was available for } 320 \text { patients in the alteplase group and } 307 \text { in the control group. }\end{array}$} \\
\hline
\end{tabular}

adjusted OR $5 \cdot 58[1 \cdot 22-25 \cdot 50] ; \mathrm{p}=0 \cdot 024)$. More cases of radiologically defined parenchymal haemorrhage type 2 were seen in the alteplase group than in the control group (11 [3\%] vs three [1\%], adjusted OR $3 \cdot 51$ [0.98-12.60]; $\mathrm{p}=0 \cdot 068)$.

A sensitivity analysis excluding THAWS, being the only trial that used a lower dose of alteplase, revealed similar findings with a significant benefit of alteplase for the primary endpoint and secondary efficacy endpoints, despite a non-significant higher number of deaths with alteplase (appendix p 18).

Prespecified subgroup analyses for the primary outcome are shown in figure 2. No evidence was seen of significant heterogeneity of the treatment effect across any of the following variables: dose of alteplase $(0.9$ vs $0.6 \mathrm{mg} / \mathrm{kg}$ bodyweight), age ( $\leq 60$ years vs $>60$ years), sex, baseline stroke severity (NIHSS $\leq 10$ vs $>10$ ), any vessel occlusion, large vessel occlusion, imaging 


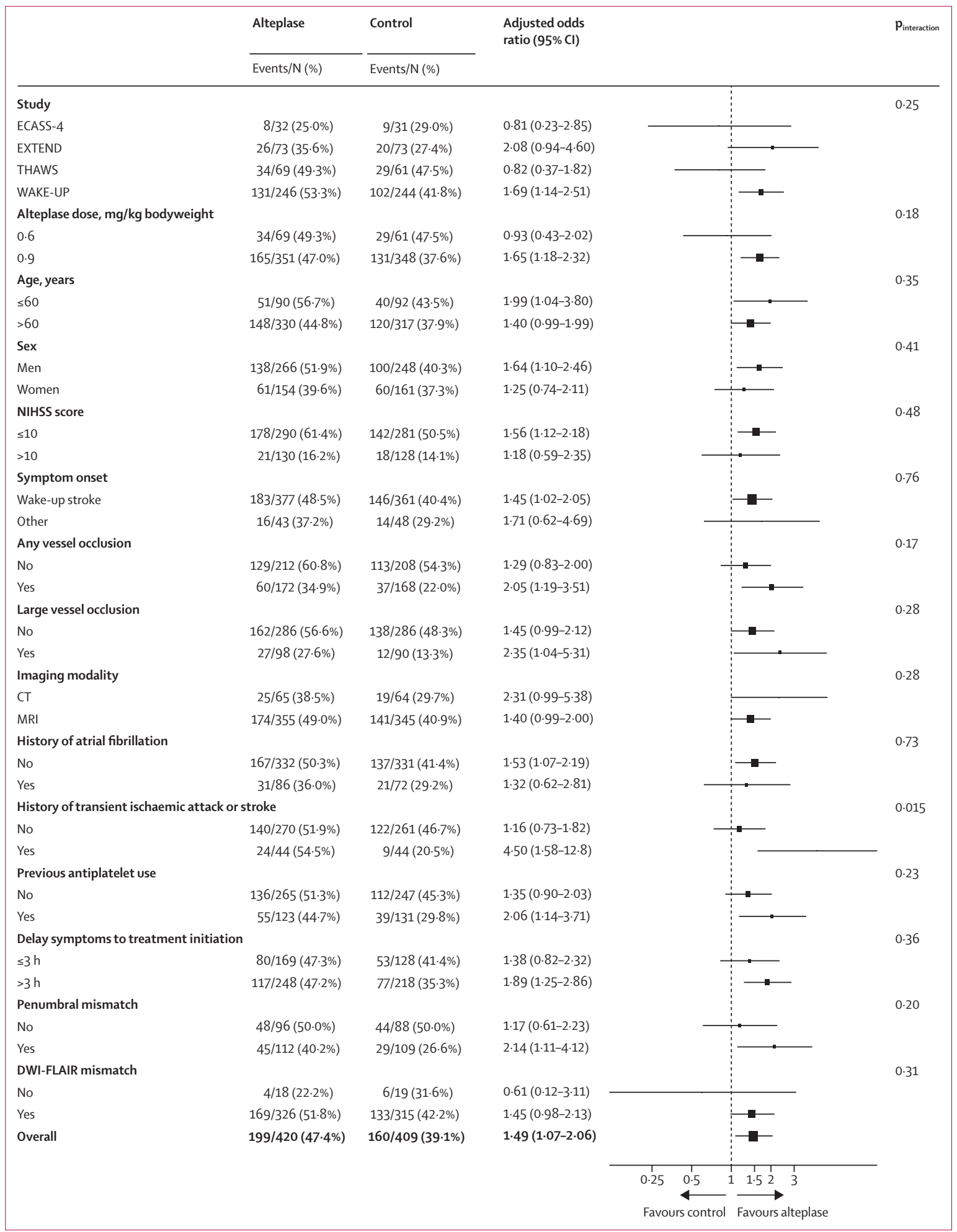

Figure 2: Subgroup analyses

Forest plots for the primary outcome of favourable outcome (modified Rankin Scale 0-1 at 90 days) in all patients for predefined subgroups. DWI-FLAIR=diffusion weighted imaging-fluid attenuated inversion recovery. NIHSS=National Institute of Health Stroke Scale. 
modality (CT vs MRI), history of atrial fibrillation, previous antiplatelet use, delay from symptom recognition to treatment ( $\leq 3 \mathrm{~h} v s>3 \mathrm{~h}$ ), penumbral mismatch present, or DWI-FLAIR mismatch present. Significant heterogeneity of treatment effect was observed for history of transient ischaemic attack or stroke with larger benefit in the subgroup of patients with history of stroke and transient ischaemic attack $\left(\mathrm{p}_{\text {interaction }}=0 \cdot 015\right)$.

Subgroup analyses for secondary outcomes and the safety outcome are provided in the appendix (pp 21-24). We observed no evidence of heterogeneity of treatment effect across any of the subgroups for mortality. Due to the overall small numbers of symptomatic intracranial haemorrhage and parenchymal haemorrhage type 2 , we did not do a subgroup analysis on these two safety parameters. Adding data from the single-arm MR WITNESS trial to the analysis did not alter the main findings (appendix p 25).

\section{Discussion}

This meta-analysis of individual patient data from four randomised controlled trials showed that intravenous alteplase is beneficial in patients with stroke with unknown time of onset selected by imaging biomarkers using MRI or CT perfusion. Such patients, who had a DWI-FLAIR mismatch on MRI or a penumbral mismatch on perfusion-diffusion MRI or CT perfusion and who received intravenous alteplase had a higher likelihood of a favourable functional outcome at 90 days after stroke than controls. The prevalence of severe disability or death (mRS score 4-6) was lower with alteplase, but treatment with alteplase was associated with a small but significant increase in the prevalence of symptomatic intracranial haemorrhage and an increase in mortality. The lower number of deaths in the control group (mRS score 6) was offset by an increased proportion of bedridden or nursing home outcomes (mRS score 5) in the control group. Importantly, there was a significant net and clinically important benefit of intravenous alteplase across the entire range of functional outcomes in ordinal analysis of the mRS.

Our analysis strengthens the results of individual trials and extends the information on treatment effect in the subgroup of patients with stroke with unknown time of onset. The WAKE-UP trial exclusively randomly assigned patients with stroke with unknown time of onset and showed improved functional outcome with intravenous alteplase guided by MRI with DWI-FLAIR mismatch.? The EXTEND trial included both patients in a late time window up to $9 \mathrm{~h}$ after stroke symptom onset and those with stroke with unknown time of onset guided by penumbral imaging, and also showed a benefit of intravenous alteplase on functional outcome in these patients..$^{10}$ Pooling individual data from only the patients with stroke with unknown time of onset from these trials and two further randomised trials applying imaging biomarker selection to enrol patients resulted in a population of 843 patients with stroke with unknown time of onset randomly assigned on the basis of advanced brain imaging. In this population, the adjusted OR for a favourable outcome with alteplase was 1.48 (95\% CI $1 \cdot 07-2 \cdot 06)$, with an absolute increase of $8 \%$ patients with favourable outcome translating into a number-needed-totreat of 12 . This treatment effect is similar to the treatment effect of intravenous alteplase within $4.5 \mathrm{~h}$ of known stroke onset, with an adjusted OR of 1.75 (95\% CI $1 \cdot 35-2 \cdot 27)$ within $3 \mathrm{~h}$, and of $1.26(95 \% \mathrm{CI} 1 \cdot 05-1 \cdot 51)$ after $3 \cdot 0-4 \cdot 5 \mathrm{~h}^{4}$ This result supports the validity and clinical utility of the concept of imaging-based selection of acute patients with stroke for reperfusion treatment in cases in which information on the time of symptom onset is not available.

The trials included in this meta-analysis differed in design and imaging inclusion criteria but they all relied on imaging biomarkers beyond non-contrast CT and vessel imaging. WAKE-UP and THAWS applied the DWI-FLAIR mismatch concept and randomly assigned patients if an MRI showed a mismatch between an acute ischaemic lesion that was visible on DWI, while there was no marked parenchymal hyperintensity on FLAIR on the basis of visual judgment, indicating an ischaemic lesion age of less than $4.5 \mathrm{~h}$ and the absence of severe and irreversible tissue damage. ${ }^{6}$ EXTEND and ECASS-4 used the concept of penumbral imaging for patient selection. They randomly assigned patients who showed a relevant amount of salvageable brain tissue defined by a limited infarct core surrounded by a larger area of critically hypoperfused tissue as shown by perfusiondiffusion MRI or CT perfusion mismatch, ${ }^{13}$ similar to the approach used to guide endovascular stroke treatment in late or unknown time windows in two trials from the past 3 years. ${ }^{11,12}$

This meta-analysis indicates that both concepts of imaging biomarker selection allow for effective identification of patients for reperfusion treatment after ischaemic stroke. Nevertheless, there are inherent advantages and disadvantages to these concepts. DWI-FLAIR mismatch requires MRI, but does not need perfusion imaging. It does not require any post-processing but is effective with simple visual judgment of routine MRI sequences with a high inter-rater agreement as compared with quantitative evaluation by measurement of FLAIR signal intensity. ${ }^{22}$ Moreover, the DWI-FLAIR mismatch allows for treatment of patients with lacunar strokes, a subgroup of the WAKE-UP trial in whom the treatment effect of alteplase was similar to patients with other subtypes of stroke. ${ }^{23}$ These patients would not have met criteria for a relevant amount of salvageable tissue in perfusion-based penumbral mismatch imaging. By contrast, penumbral mismatch might identify patients with salvageable tissue despite already marked hyperintensity on FLAIR and thus increase the number of patients given thrombolysis. ${ }^{24}$ For clinical practice, we conclude that any of the mismatch concepts are effective and can 
be recommended for guiding intravenous thrombolysis with alteplase in stroke with unknown time of onset.

Our meta-analysis showed increased numbers of symptomatic intracranial haemorrhage with alteplase treatment, which was expected based on the results of a previous pooled analysis of stroke thrombolysis trials. ${ }^{4}$ The increased risk of symptomatic intracranial haemorrhage corresponds to the biological effects of alteplase but also relates to higher rates of reperfusion. ${ }^{25}$ The overall small proportion of $3 \%$ of intracranial haemorrhages with alteplase in this population with stroke with unknown time of onset is similar to the $3.5 \%$ rate of symptomatic intracranial haemorrhage according to SITS-MOST definition in the pooled analysis of stroke thrombolysis trials in patients with known onset. ${ }^{4}$ It is reassuring that no excess of intracranial haemorrhages was observed in unknown onset strokes compared with those with known onset. We also observed an increase in mortality with alteplase treatment $(6 \%$ vs $3 \%$ in the control group), while cases of severe disability or death were lower with alteplase, and amounts of death and bedridden or nursing home outcomes were similar for both treatment groups. Slightly increased mortality is not unexpected, as a significant increase of early deaths has been reported in the previous pooled analysis of stroke thrombolysis trials, ${ }^{4}$ which at least partly can be related to an increased rate of fatal intracranial haemorrhage. In our analysis, seven (26\%) of 27 deaths in the alteplase group were attributable to symptomatic intracranial haemorrhage and thus possibly related to treatment with alteplase, whereas most deaths were considered unrelated and of non-neurological cause. The increased mortality did not negate the net benefit of intravenous alteplase, as the analysis of functional outcome across the entire range of the mRS including death showed a significant benefit with overall better functional outcome with alteplase treatment.

Our analysis did not identify a significant treatment heterogeneity in any relevant subgroups but confirmed a consistent treatment effect across a wide range of subgroups. Subgroup analyses also revealed no interaction of treatment effect with vessel occlusion. We also observed a significant treatment benefit in the subgroup of patients with a large vessel occlusion with an adjusted OR of $2 \cdot 35$ (95\% CI 1.04-5.32). This finding is of clinical importance and reinforces the finding in the previous perfusion mismatch meta-analysis of a clear benefit of alteplase in large vessel occlusion. ${ }^{13}$ Doubts concerning the efficacy of intravenous alteplase in large vessel occlusion together with an assumed increased risk for intracranial haemorrhage have led to questioning the rationale for intravenous thrombolysis in these patients before thrombectomy. ${ }^{26}$ The results of our pooled analysis support treatment with alteplase in patients with large vessel occlusion and stroke with an unknown time of onset, especially if patients present to centres in which thrombectomy is not immediately available.
Our meta-analysis has limitations. We cannot draw any inference on possible effects of the different dose of alteplase, as the THAWS trial was the only trial that used the lower dose of $0.6 \mathrm{mg} / \mathrm{kg}$ bodyweight. Thus, we cannot separate a possible interaction of treatment effect with alteplase dose from overall trial effects. This consideration is even more important, as the THAWS trial differed from the others in that it was the only trial that was not placebo-controlled but open-label and, resulting from this design, allowed for early use of antithrombotic medication in the control group. Beyond early termination and the trial being underpowered, these factors might have been reasons why this individual trial had neutral outcomes. ${ }^{18}$ Moreover, all four randomised trials were terminated early, either due to cessation of funding (WAKE-UP), new evidence (EXTEND, THAWS), or change of clinical practice (ECASS-4). All of these are external reasons, thus, the potential bias is low, but early termination resulted in an overall smaller number of patients available for this individual patient data meta-analysis. With a sample size of 843 patients, statistical power to provide adjusted treatment-effect estimates for smaller subgroups was still limited, as reflected by wide $95 \%$ CIs for some of the subgroup analyses. As most patients included in this meta-analysis had rather mild to moderate strokes with a median NIHSS score of 7 , results might not be generalisable to patients with severe stroke and large core. Finally, although we observed no heterogeneity of treatment effect between the trials, we have to consider that the results of the meta-analysis are to some extent driven by the WAKE-UP trial, representing almost $60 \%$ of the patients included in the analysis.

The requirement for advanced imaging beyond noncontrast CT and vessel imaging-ie, either perfusion CT or MRI, might still represent a potential limitation for implementation of the studied treatment approach in some regions or hospitals. However, given the evidence from WAKE-UP and EXTEND, together with the evidence for effective imaging-guided endovascular stroke treatment in an extended or unknown time window, advanced brain imaging has to be considered a requirement for providing state of the art evidence-based stroke treatment. ${ }^{11,12}$ The results of this meta-analysis should further support efforts to make these necessary imaging techniques more widely available, so that access to this effective treatment is available to as many patients with stroke as possible.

In conclusion, intravenous alteplase improved functional outcome in patients with stroke of unknown onset selected by imaging biomarkers from MRI or CT perfusion. A net benefit was observed across the entire range of functional outcome despite an increased risk of symptomatic intracranial haemorrhage and higher mortality with alteplase. Treatment benefit was consistent across a wide range of subgroups, including patients with large vessel occlusion. This individual patient data meta-analysis extends the evidence from individual trials 
and supports the use of imaging biomarkers to guide treatment with intravenous alteplase in patients with stroke with an unknown time of onset.

\section{Contributors}

GT, FB, HM, PAR, KT, MK, WH, SMD, GAD, and CG developed the study protocol, interpreted the data, and drafted the manuscript. FB analysed the data. FB and GT accessed and verified the underlying data, and all authors had access to the data and accept responsibility for submitting the Article for publication. All authors collected data and edited the manuscript.

\section{Declaration of interests}

FB reports grants from University Medical Center Hamburg-Eppendorf, during the conduct of the study. MB reports personal fees from Boehringer Ingelheim, Merck, Bayer, Teva, BBraun, Vascular Dynamics, and Grifols; grants and personal fees from Novartis and Guerbet, grants from Siemens, Hopp Foundation, Deutsche Forschungsgemeinschaft, the EU, and Stryker, outside of the submitted work. SMD reports personal fees from Abbott, Boehringer Ingelheim, Bayer, Tide Pharmaceuticals, and Medtronic; and grants from the National Health and Medical Research Council of Australia, outside of the submitted work. GAD reports grants from National Health and Medical Research Council Australia, during the conduct of the study; personal fees from Allergan, Amgen, Bayer, Boehringer Ingelheim, Pfizer, Servier, outside of the submitted work; MEn reports grants from the EU, during the conduct of the study; grants and fees paid to the institution from Bayer, fees paid to the institution from Boehringer Ingelheim, Bristol Myers Squibb, Pfizer, Daiichi Sankyo, Amgen, Sanofi, Novartis, GSK, and Covidien, outside of the submitted work. JBF reports personal fees from BioClinica, EISAI, Artemida, Biogen, Bristol Myers Squibb, Brainomix, and Cerevast, outside of the submitted work. CG reports personal fees from Amgen, Bayer vital, Bristol Myers Squibb, Boehringer Ingelheim, Sanofi Aventis, Abbott, and Prediction Biosciences, outside of the submitted work. WH reports grants from Boehringer Ingelheim. MK reports personal fees from Bayer Yakuhin, Bristol Myers Squibb and Pfizer, Otsuka, Daiichi-Sankyo, Nippon Boehringer Ingelheim, Takeda, and Ono, grants from Takeda, Daiichi-Sankyo, Nippon Boehringer Ingelheim, Astellas, Pfizer, and Shionogi, outside of the submitted work. RL reports fees paid to the institution from Bayer, Boehringer Ingelheim, Genentech, Ischemaview, Medtronic, and Occlutec, outside of the submitted work. DL reports grants from Pfizer, fees paid to the institution from European Stroke Organisation and John Wiley \& Sons; and grants from Bayer and Boehringer Ingelheim, outside of the submitted work. KWM reports personal fees and non-financial support from Boehringer Ingelheim, personal fees from Bayer, Daiichi-Sankyo, ReNeuron, and Biogen, outside the scope of the submitted work. MI reports personal fees from Daiichi Sankyo, Bayer, Bristol Myers Squibb, and Medtronic, outside of the submitted work. MWP reports grants from National Health and Medical Research Council of Australia, personal fees from Medtronic advisory board and Boehringer Ingelheim advisory board, outside of the submitted work. PR reports grants from Boehringer Ingelheim, during the conduct of the study; personal fees from Bayer, Boehringer Ingelheim, Pfizer, and Daichii Sankyo, outside of the submitted work. PDS reports personal fees from Boehringer Ingelheim, Bristol Myers Squibb and Pfizer, Daiichi Sankyo, Portola, AstraZeneca, and AbbVie, outside of the submitted work. SSS reports grants from National Institutes of Health (NIH), during the conduct of the study. LHS reports grants from National Institute of Neurological Disorders and Stroke (NINDS) and Genentech, during the conduct of the study; grants from NINDS, personal fees from Genentech, Medtronic, and LifeImage, outside of the submitted work; and serves on data safety monitoring boards for Penumbra, Diffusion Pharma, and the Charite Hospital B_PROUD trial of mobile stroke units. CZS reports grants from Novo Nordisk Foundation, during the conduct of the study. VT reports grants from the EU, during the conduct of the study; personal fees from Boehringer Ingelheim, Bayer, Pfizer and Bristol Myers Squibb, Medtronic, Amgen, and Allergan, outside of the submitted work. GT reports grants and personal fees from Bayer, personal fees from Acandis, Boehringer Ingelheim, Bristol Myers Squibb and Pfizer, Daiichi Sankyo, Portola, and Stryker, outside of the submitted work. DT reports personal fees from Abbott, Bayer,
Boehringer Ingelheim, Daiichi Sankyo, Medtronic, and Pfizer, outside of the submitted work. KT reports grants from Japan Agency for Medical Research and Development, during the conduct of the study; personal fees from Daiichi-Sankyo, Bayer Yakuhin, Bristol Myers Squibb, and Nippon Boehringer Ingelheim, outside of the submitted work. NW reports grants from Boehringer-Ingelheim, during the conduct of the study. SW reports grants from NIH Grant P50NS051343, during the conduct of the study; personal fees from Genentech, outside of the submitted work. OW reports grants from NIH; grants and non-financial support from Genentech, during the conduct of the study; personal fees from Penumbra and Genentech, outside of the submitted work. OW has a patent "Delay-compensated calculation of tissue blood flow,"

(US Patent 7,512,435. March 31, 2009) with royalties paid to General Electric, Siemens, Olea Medical, and Imaging Biometrics. All other authors declare no competing interests.

\section{Data sharing}

Individual participant data that underlie the results reported in this Article will be made available on request, after de-identification, beginning 6 months and ending 24 months after Article publication to investigators whose proposed use of the data have been approved by the EOS steering committee.

\section{Acknowledgments}

WAKE-UP was supported by a grant (number 278276) from the EU Seventh Framework programme. EXTEND was funded by the National Health and Medical Research Council, an Australian Government organisation and Commonwealth Scientific and Industrial Research Organisation. Boehringer Ingelheim provided the study investigational products free of charge. THAWS was supported by the Japan Agency for Medical Research and Development (AMED; 19ek0210091h0003 and 19lk0201094h0001), and the Ministry of Health, Labour, and Welfare, and the Mihara Cerebrovascular Disorder Research Promotion Fund. ECASS-4 was an investigator-initiated trial supported by an unrestricted grant from Boehringer Ingelheim (Germany). MR WITNESS was supported by the NIH National Institute of Neurological Disorders and Stroke (NINDS) Specialized Program of Transitional Research in Acute Stroke (SPOTRIAS; P50-NS051343) and NINDS Division of Intramural Research, was done in part at the Athinoula A Martinos Center for Biomedical Imaging at Massachusetts General Hospital, using resources provided by the Center for Functional Neuroimaging Technologies (P41EB015896), a P41 Biotechnology Resource Grant supported by the NIH National Institute of Biomedical Imaging and Bioengineering. Genentech provided alteplase free of charge to the study for distribution to all sites except to the NINDS intramural branch and starting in year 2 provided supplemental site payments to permit expansion to 14 sites.

\section{References}

1 Lees KR, Bluhmki E, von Kummer R, et al. Time to treatment with intravenous alteplase and outcome in stroke: an updated pooled analysis of ECASS, ATLANTIS, NINDS, and EPITHET trials. Lancet 2010; 375: 1695-703.

2 National Institute of Neurological Disorders and Stroke rt-PA Stroke Study Group. Tissue plasminogen activator for acute ischemic stroke. N Engl J Med 1995; 333: 1581-87.

3 Hacke W, Kaste M, Bluhmki E, et al. Thrombolysis with alteplase 3 to 4.5 hours after acute ischemic stroke. N Engl J Med 2008; 359: 1317-29.

4 Emberson J, Lees KR, Lyden P, et al. Effect of treatment delay, age, and stroke severity on the effects of intravenous thrombolysis with alteplase for acute ischaemic stroke: a meta-analysis of individual patient data from randomised trials. Lancet 2014; 384: 1929-35.

5 Rimmele DL, Thomalla G. Wake-up stroke: clinical characteristics, imaging findings, and treatment option-an update. Front Neurol 2014; 5: 35.

6 Thomalla G, Cheng B, Ebinger M, et al. DWI-FLAIR mismatch for the identification of patients with acute ischaemic stroke within $4.5 \mathrm{~h}$ of symptom onset (PRE-FLAIR): a multicentre observational study. Lancet Neurol 2011; 10: 978-86.

7 Thomalla G, Simonsen CZ, Boutitie F, et al. MRI-guided thrombolysis for stroke with unknown time of onset. $N$ Engl J Med 2018; 379: 611-22. 
8 Powers WJ, Rabinstein AA, Ackerson T, et al. Guidelines for the early management of patients with acute ischemic stroke: 2019 update to the 2018 guidelines for the early management of acute ischemic stroke: a guideline for healthcare professionals from the American Heart Association/American Stroke Association. Stroke 2019; 50: e344-418.

9 Ahmed N, Audebert H, Turc G, et al. Consensus statements and recommendations from the ESO-Karolinska Stroke Update Conference, Stockholm 11-13 November 2018. Eur Stroke J 2019; 4: 307-17.

10 Ma H, Campbell BCV, Parsons MW, et al. Thrombolysis guided by perfusion imaging up to 9 hours after onset of stroke. $N$ Engl J Med 2019; 380: 1795-803.

11 Albers GW, Marks MP, Kemp S, et al. Thrombectomy for stroke at 6 to 16 hours with selection by perfusion imaging. $N$ Engl J Med 2018; 378: 708-18.

12 Nogueira RG, Jadhav AP, Haussen DC, et al. Thrombectomy 6 to 24 hours after stroke with a mismatch between deficit and infarct. $N$ Engl J Med 2018; 378: 11-21.

13 Campbell BCV, Ma H, Ringleb PA, et al. Extending thrombolysis to 4.5-9 h and wake-up stroke using perfusion imaging: a systematic review and meta-analysis of individual patient data. Lancet 2019; 394: 139-47.

14 Wahlgren N, Ahmed N, Dávalos A, et al. Thrombolysis with alteplase for acute ischaemic stroke in the safe implementation of thrombolysis in stroke-monitoring study (SITS-MOST): an observational study. Lancet 2007; 369: 275-82.

15 von Kummer R, Broderick JP, Campbell BC, et al. The Heidelberg Bleeding Classification: classification of bleeding events after ischemic stroke and reperfusion therapy. Stroke 2015; 46: 2981-86.

16 Thomalla G, Fiebach JB, Østergaard L, et al. A multicenter, randomized, double-blind, placebo-controlled trial to test efficacy and safety of magnetic resonance imaging-based thrombolysis in wake-up stroke (WAKE-UP). Int J Stroke 2014; 9: 829-36.

17 Higgins JP, Thompson SG. Quantifying heterogeneity in a metaanalysis. Stat Med 2002; 21: 1539-58.
18 Koga M, Yamamoto H, Inoue M, et al. Thrombolysis with alteplase at $0.6 \mathrm{mg} / \mathrm{kg}$ for stroke with unknown time of onset: a randomized controlled trial. Stroke 2020; 51: 1530-38.

19 Ringleb P, Bendszus M, Bluhmki E, et al. Extending the time window for intravenous thrombolysis in acute ischemic stroke using magnetic resonance imaging-based patient selection. Int J Stroke 2019; 14: 483-90.

20 Toyoda K, Koga M, Iguchi Y, et al. Guidelines for intravenous thrombolysis (recombinant tissue-type plasminogen activator), the third edition, March 2019: a guideline from the Japan Stroke Society. Neurol Med Chir 2019; 59: 449-91.

21 Schwamm LH, Wu O, Song SS, et al. Intravenous thrombolysis in unwitnessed stroke onset: MR WITNESS trial results. Ann Neurol 2018; 83: 980-93.

22 Galinovic I, Puig J, Neeb L, et al. Visual and region of interest-based inter-rater agreement in the assessment of the diffusion-weighted imaging- fluid-attenuated inversion recovery mismatch. Stroke 2014 45: 1170-72.

23 Barow E, Boutitie F, Cheng B, et al. Functional outcome of intravenous thrombolysis in patients with lacunar infarcts in the WAKE-UP Trial. JAMA Neurol 2019; 76: 641-49.

24 Scheldeman L, Wouters A, Boutitie F, et al. Different mismatch concepts for magnetic resonance imaging-guided thrombolysis in unknown onset stroke. Ann Neurol 2020; 87: 931-38.

25 Thomalla G, Sobesky J, Köhrmann M, et al. Two tales: hemorrhagic transformation but not parenchymal hemorrhage after thrombolysis is related to severity and duration of ischemia: MRI study of acute stroke patients treated with intravenous tissue plasminogen activator within 6 hours. Stroke 2007; 38: 313-18.

26 Fischer U, Kaesmacher J, Mendes Pereira V, et al. Direct mechanical thrombectomy versus combined intravenous and mechanical thrombectomy in large-artery anterior circulation stroke: a topical review. Stroke 2017; 48: 2912-18 